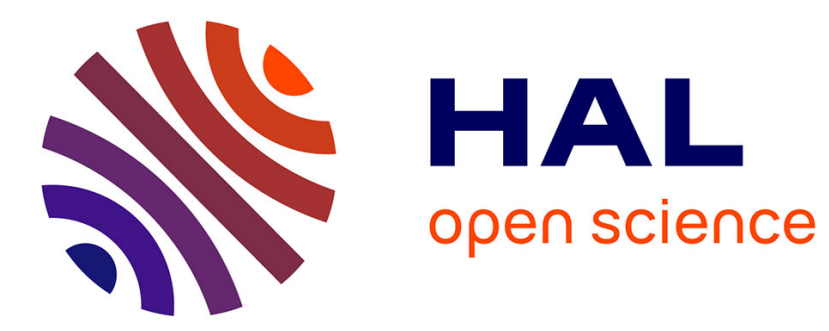

\title{
Spectrally efficient multiplexing of chaotic light
}

\author{
Damien Rontani, A. Locquet, M. Sciamanna, D. S Citrin
}

\section{To cite this version:}

Damien Rontani, A. Locquet, M. Sciamanna, D. S Citrin. Spectrally efficient multiplexing of chaotic light. Optics Letters, 2010, 35 (12), pp.2016-2018. 10.1364/OL.35.002016 . hal-03079747

\section{HAL Id: hal-03079747 \\ https://hal.science/hal-03079747}

Submitted on 17 Dec 2020

HAL is a multi-disciplinary open access archive for the deposit and dissemination of scientific research documents, whether they are published or not. The documents may come from teaching and research institutions in France or abroad, or from public or private research centers.
L'archive ouverte pluridisciplinaire HAL, est destinée au dépôt et à la diffusion de documents scientifiques de niveau recherche, publiés ou non, émanant des établissements d'enseignement et de recherche français ou étrangers, des laboratoires publics ou privés. 


\title{
Spectrally Efficient Multiplexing of Chaotic Light
}

\author{
D. Rontani ${ }^{1,2,3 *}$, A. Locquet $^{1,3}$, M. Sciamanna ${ }^{2,1}$, and D.S. Citrin ${ }^{1,3}$ \\ ${ }^{1}$ UMI 2958 Georgia Tech-CNRS, GeorgiaTech Lorraine, 2-3 Rue Marconi F-57070 Metz, France \\ ${ }^{2}$ Supélec, OPTEL and LMOPS EA-4423, 2 Rue Edouard Belin F-57070 Metz, France \\ ${ }^{3}$ Georgia Institute of Technology, School of Electrical and Computer Engineering, Atlanta, Georgia 30324-0250, USA \\ ${ }^{*}$ Corresponding author: damien.rontani@supelec.fr
}

Compiled April 4, 2010

We numerically demonstrate multiplexing and demultiplexing of two distinct chaotic optical signals with strongly overlapped spectra, which are generated by mutually coupled external-cavity semiconductor lasers. Demultiplexing is performed by complete chaos synchronization, while the lasers at the receiving end are optically injected with the same multiplexed signal that couples the emitters together. Such a configuration could lead to spectrally efficient chaos-based optical encryption and decryption of multiple data streams. (C) 2010 Optical Society of America

OCIS codes: $140.1540,190.3100,060.4230$

Optical chaos-based communication between two users has attracted considerable attention since high-bit-rate physical layer security can be achieved $[1,2]$. When more than two users are involved, and a single optical channel is available, it is necessary to multiplex the chaotic signals of the different users. In conventional optical communications, time- and wavelength-division multiplexing (TDM and WDM) are well known protocols that make use of different time slots and wavelengths, respectively, to convey each user's signal. It has been proposed to apply WDM [3-8] to optical chaotic communications. Chaotic WDM can be achieved using either multiple chaotic single-mode lasers operating at detuned wavelengths [3-5] or multi-mode lasers [6-8]. Users on the receiving end synchronize their chaotic fluctuations with those of the same-frequency emitter. Though interesting, chaotic WDM has the disadvantage of requiring a large frequency separation between channels to avoid interference between each user's wide spectrum [5]. The application of the other standard multiplexing approach, TDM, would not lead to any improvement of the spectral efficiency of the communication.

In this letter, we show how to use more efficiently the large bandwidth of optical chaos for multiplexed communications. For this purpose, we propose an optical analogy of active-passive decomposition (APD) [9] to create a multiplexed signal that equally drives the dynamics of the master and slave lasers. The separation of each carrier is performed through complete synchronization of each master/slave laser pair. The synchronization properties are numerically investigated in the special case of two pairs of lasers.

Figure 1 shows a two-user setup composed of two mutually coupled master lasers $\left(\mathrm{M}_{1}\right.$ and $\left.\mathrm{M}_{2}\right)$ unidirectionally coupled to two slave lasers $\left(\mathrm{S}_{1}\right.$ and $\left.\mathrm{S}_{2}\right)$. Each master is subjected to delayed optical feedback from mirror $\mathrm{M}_{f}$ and to delayed optical injection from the other master. A linear combination of the two masters' delayed complex optical fields is thus injected in each master, but with

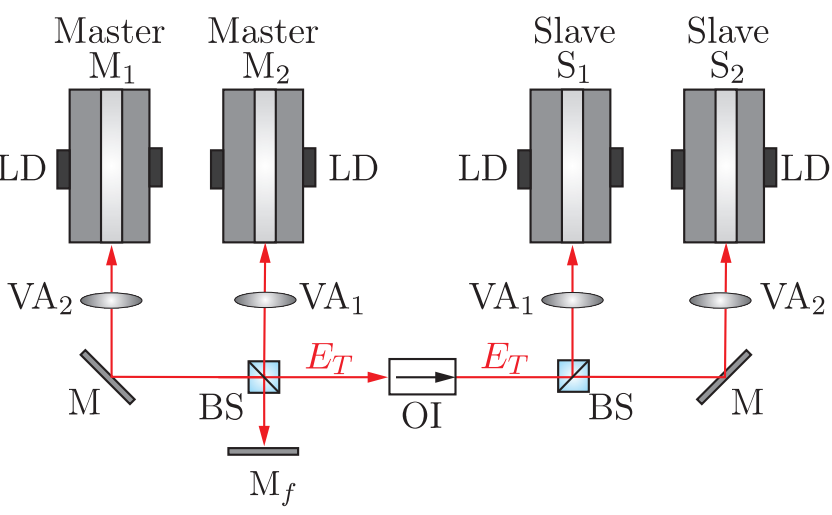

Fig. 1. (Color online) Multiplexing scheme based on optically coupled semiconductor lasers. LD: laser diode (labeled M1, M2 for the masters and S1, S2 for the slaves), CS: current source, $\mathrm{M}, \mathrm{M}_{f}$ : mirrors, $\mathrm{VA}_{1}, \mathrm{VA}_{2}$ : variable attenuators, BS: 50/50 beam splitter, OI: optical isolator. $E_{T}$ is the multiplexed field sent to the slave lasers.

specific strength (different variable attenuators), phase, and delay (different optical paths) for each field. The same linear combinations are then optically injected, after propagation on a free-space optical channel, into the uncoupled slave semiconductor lasers $\left(\mathrm{S}_{1}\right.$ and $\left.\mathrm{S}_{2}\right)$. As a result, the dynamics of each slave is driven by an identical, though time-shifted, copy of the signal that drives the corresponding master. This coupling architecture can be considered an application of APD to multi-user optical delayed systems.

We have investigated theoretically the synchronization properties of our setup based on the assumption of single-mode semiconductor lasers modeled by the LangKobayashi equations [11]. The system of equations for $n$ 

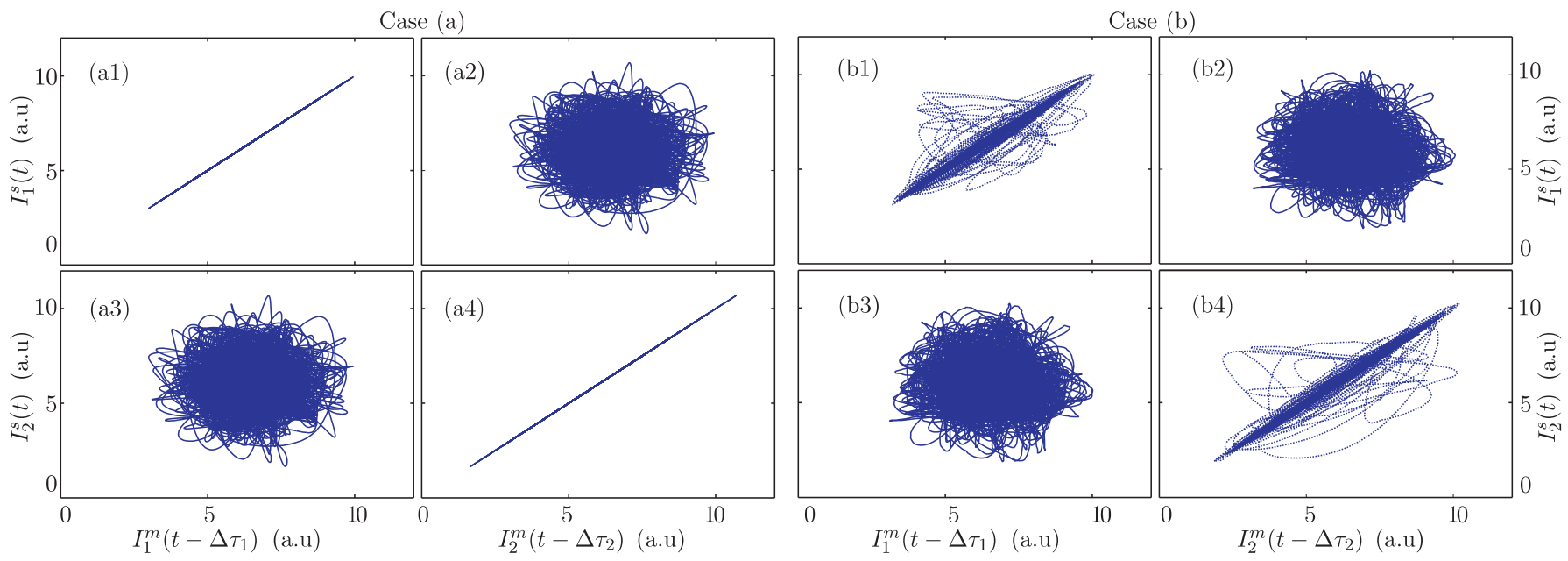

Fig. 2. (Color online) Synchronization diagrams in planes $\left(I_{k=1,2}^{m}\left(t-\Delta \tau_{k}\right), I_{j=1,2}^{s}(t)\right)$ without (in case (a)) and with spontaneous-emission noise (in case (b), $\beta_{s p}=1000 \mathrm{~s}^{-1}$ ). The operational parameters are $J_{1}^{m}=J_{1}^{s}=2.75 J_{t h}$,

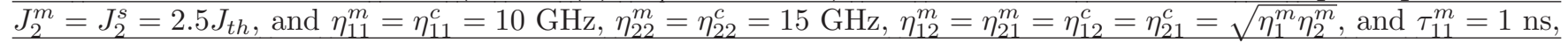
$\tau_{22}^{m}=4 \mathrm{~ns}, \quad \tau_{11}^{c}=10 \mathrm{~ns}, \quad \tau_{22}^{c}=15 \mathrm{~ns}$, and $\Delta \omega_{11}^{m / s}=\Delta \omega_{22}^{m / s}=\Delta \omega_{12}^{m / m}=0 \mathrm{GHz}$. The internal parameters are: $\underline{\alpha}_{1}^{m, s}=5, \alpha_{2}^{m, s}=4, \tau_{p 1}^{m, s}=2 \mathrm{ps}, \tau_{p 2}^{m, s}=1 \mathrm{ps}, \gamma_{s 1}^{m, s}=0.5 \mathrm{GHz}, \gamma_{s 2}^{m, s}=1 \mathrm{GHz}, \varepsilon_{1}^{m, s}=5 \times 10^{-7}, \varepsilon_{2}^{m, s}=2.5 \times 10^{-7}$,

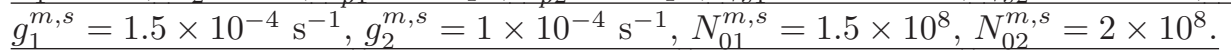

emitter-receiver laser pairs reads

$$
\begin{aligned}
\frac{d E_{k}^{m}}{d t} & =\frac{1}{2}\left(1+i \alpha_{k}^{m}\right)\left(G_{k}^{m}-1 / \tau_{p k}^{m}\right) E_{k}^{m}+F_{k}^{m} \\
& +\sum_{j=1}^{n} \eta_{j k}^{m} \exp \left(-i \omega_{0 j}^{m} \tau_{j k}^{m}+i \Delta \omega_{j k}^{m / m} t\right) E_{j}^{m}\left(t-\tau_{j k}^{m}\right) \\
\frac{d E_{k}^{s}}{d t} & =\frac{1}{2}\left(1+i \alpha_{k}^{s}\right)\left(G_{k}^{s}-1 / \tau_{p k}^{s}\right) E_{k}^{s}+F_{k}^{s} \\
& \left.\left.+\sum_{j=1}^{n} \eta_{j k}^{c} \exp \left(-i \omega_{0 j}^{s} \tau_{j k}^{c}+i \Delta \omega_{j k}^{m / s} t\right) E_{j}^{m}\left(t-\tau_{j k}^{q}\right)^{2}\right)\right) \\
\frac{d N_{k}^{m, s}}{d t} & =J_{k}^{m, s}-\gamma_{s k}^{m, s} N_{k}^{m, s}-G_{k}^{m, s}\left|E_{k}^{m, s}\right|^{2}
\end{aligned}
$$

where the subscript $k$ denotes the $k$ th lasers pair and superscripts $m, s$ master or slave variables, respectively, $E_{k}^{m, s}=\left|E_{k}^{m, s}\right| e^{i \phi_{k}^{m, s}}$ is the slowly varying complex electric field, $N_{k}^{m, s}$ the carrier number, $G_{k}^{m, s}=g_{k}^{m, s}\left(N_{k}^{m, s}-\right.$ $\left.N_{0 k}^{m, s}\right) /\left(1+\varepsilon_{k}^{m, s}\left|E_{k}^{m, s}\right|^{2}\right)$ is the nonlinear gain with $g_{k}^{m, s}$ the differential gain, $N_{0 k}^{m, s}$ the carrier number at transparency, $\varepsilon_{k}^{m, s}$ the gain saturation coefficient, $\tau_{p k}^{m, s}$ the photon lifetime, $\alpha_{k}^{m, s}$ is the linewidth enhancement factor, $\gamma_{s k}^{m, s}$ is the carrier decay rate, $J_{k}^{m, s}$ is the pumping current density, $\omega_{0 k}^{m, s}$ is the free-running laser frequency, $\tau_{j k}^{m}\left(\tau_{j k}^{c}\right), \eta_{j k}^{m}\left(\eta_{j k}^{c}\right), \Delta \omega_{j k}^{m / m}=\omega_{0 j}^{m}-\omega_{0 k}^{m}\left(\Delta \omega_{j k}^{m / s}=\right.$ $\left.\omega_{0 j}^{m}-\omega_{0 k}^{s}\right)$ are the flight time, injection strength, and detuning between the $j$ th and the $k$ th master laser (the $j$ th master laser and the $k$ th slave laser). Spontaneous emission noise is modeled by Langevin sources $F_{k}^{m, s}=$ $\sqrt{2 \beta_{k}^{m, s} N_{k}^{m, s}} \xi_{k}^{m, s}$ with $\beta_{s p}$ the spontaneous emission rate and $\xi_{k}^{m, s}$ independent Gaussian white noises with unitary variance. The geometry of our system leads, with the assumption of identical perfectly reflecting mirrors and identical optical coupling efficiencies in all laser cavities, to the following relations between flight times and coupling strengths: $\tau_{j k}^{m}=\tau_{k j}^{m}=\tau_{j j}^{m}+\Delta \tau_{k j}^{m} / 2$ with $\Delta \tau_{j k}^{m}=-\Delta \tau_{k j}^{m}=\tau_{j j}^{m}-\tau_{k k}^{m}$ and $\eta_{k j}^{m}=\eta_{j k}^{m}=\sqrt{\eta_{k k}^{m} \eta_{j j}^{m}}$. Additionally, our scheme has been devised in such a ) way that in each pair $\mathrm{M}_{k} / \mathrm{S}_{k}$ is subjected to all the master chaotic optical fields $E_{j}^{m}$ with identical relative time shifts. Mathematically, this means that $\tau_{k k}^{m}-\tau_{j k}^{m}=$ $\tau_{k k}^{c}-\tau_{j k}^{c}$. Furthermore, it is necessary to set $\eta_{j k}^{m}=\eta_{j k}^{c}$ to ensure that each coupling field has the same weight in the master and slave equations within a pair. This necessary condition naturally extends the coupling requirement for complete chaos synchronization between a single master (3) and a single open-loop slave laser [12]. Under the conditions above, a regime of complete synchronization exists in each pair $\mathrm{M}_{k} / \mathrm{S}_{k}$, in the absence of noise and of parameter mismatch between the lasers of a pair, and are mathematically described by the following equations: $E_{k}^{s}(t)=$ $E_{k}^{m}\left(t-\Delta \tau_{k}\right), \phi_{k}^{s}(t)=\phi_{k}^{m}\left(t-\Delta \tau_{k}\right)-\omega_{0 k}^{m} \Delta \tau_{k}(\bmod 2 \pi)$, and $N_{k}^{s}=N_{k}^{m}\left(t-\Delta \tau_{k}\right)$, where $\Delta \tau_{k}$ is a time lag given by $\Delta \tau_{k}=\tau_{k k}^{c}-\tau_{k k}^{m}$.

Figure 2 presents theoretical synchronization diagrams of our system described by Eqs. (1)-(3) with $n=2$. They represent the evolution of the optical intensity, defined by $I_{k}^{m, s}=\left|E_{k}^{m, s}\right|^{2}$, of each master as a function of its respective slave. They are plotted without and with spontaneous noise emission in Fig. 2-case (a) and -case (b), respectively. Additionally, the synchronization quality is evaluated with the cross-correlation coefficient

$$
C_{j k}(\tau)=\frac{\left\langle\left[I_{j}^{m}(t+\tau)-\left\langle I_{j}^{m}\right\rangle\right]\left[I_{k}^{s}(t)-\left\langle I_{k}^{s}\right\rangle\right]\right\rangle}{\left[\left\langle I_{j}^{m}(t+\tau)-\left\langle I_{j}^{m}\right\rangle\right\rangle\left\langle I_{k}^{s}(t)-\left\langle I_{k}^{s}\right\rangle\right]^{1 / 2}\right.},
$$

where $\langle\cdot\rangle$ is the time-averaging operator. In Figs. 2(a1)-(a4), straight lines are observed and correspond to 
perfect chaos synchronization of each laser pair $\mathrm{M}_{1} / \mathrm{S}_{1}$ and $\mathrm{M}_{2} / \mathrm{S}_{2}\left[C_{11}\left(\Delta \tau_{1}\right)=C_{22}\left(\Delta \tau_{2}\right)=1\right]$ with time lags $\Delta \tau_{1}=9 \mathrm{~ns}$ and $\Delta \tau_{2}=11 \mathrm{~ns}$. Figures 2(a1)-(a4) display a cloud of points which reveals a low degree of correlation between each laser pair $\left[C_{12}\left(\Delta \tau_{1}-\Delta \tau_{2}\right) \approx 0.04\right]$. We find that the degree of decorrelation between each pair strongly depends on the value of coupling parameters, as will be detailed elsewhere. The introduction of noise destroys the perfect synchronization $\left[C_{11}\left(\Delta \tau_{1}\right) \approx\right.$ $C_{22}\left(\Delta \tau_{2}\right) \approx 0.97$ and $\left.C_{12}\left(\Delta \tau_{1}-\Delta \tau_{2}\right) \approx 0.07\right]$, but the trajectories in the plane $\left(I_{k}^{m}, I_{k}^{s}\right)$ remain sufficiently close to the noiseless synchronization manifolds [Fig. 2(b1)(b4)] for our scheme to be used for communication applications.

We determine that perfect synchronization remains robust to parameter mismatch between lasers in a pair as well if the mismatch level is comparable to that encountered in single emitter/receiver laser configurations [13]. Additionally, it must be noted that our APD-based coupling configuration does not limit the amount of mismatch between two different pairs (so long as they remain in chaotic regimes). By way of illustration, the case of two laser pairs has been presented, but this number can be increased: if each new master laser shares the original external cavity, and each slave laser remains decoupled and properly injected. Simulations suggest that complete synchronization still exists for a large range of coupling parameters and remains stable as $n$ is increased. This architecture has thus the potential to handle chaos-based communications between numerous users.

Finally, we investigate the spectral efficiency of the proposed multiplexing scheme by computing the optical spectrum of each master laser $\mathrm{M}_{k}$ and of the optical field $E_{T}$ propagated on the transmission channel. Figure 3 shows the spectra of the two free-running master lasers (inset) and of the coupled external-cavity master lasers. The free-running spectra are centered at $\Delta f=0 \mathrm{GHz}$. The mutual coupling and feedback induce chaotic behaviors of both $\mathrm{M}_{1}$ and $\mathrm{M}_{2}$ resulting in spectral broadening. The two chaotic spectra significantly overlap and have identical bandwidth (taken $20 \mathrm{~dB}$ below the spectrum's maximum) $\Delta f_{E_{1}^{m}} \approx \Delta f_{E_{2}^{m}} \approx 25 \mathrm{GHz}$. Since the transmitted signal $E_{T}$ has a bandwidth $\Delta f_{E_{T}} \approx 25 \mathrm{GHz}$, it presents similar spectral properties to each master chaotic optical field but with more optical power [Fig. 3]. Assuming each master signal $E_{k}^{m}(k=1,2)$ encodes a different information-bearing message, the message bit rate will be double compared to the single-ECL emitter case, while using a similar bandwidth. Our scheme could thus lead to a dramatic increase of the spectral efficiency of chaotic communications. Additionally, the transmitted signal $E_{T}$ may also mislead an eavesdropper trying to attack the system, who could consider it is as resulting from a single chaotic external-cavity semiconductor laser.

In this letter, we have shown for the first time to our knowledge the possibility to multiplex and

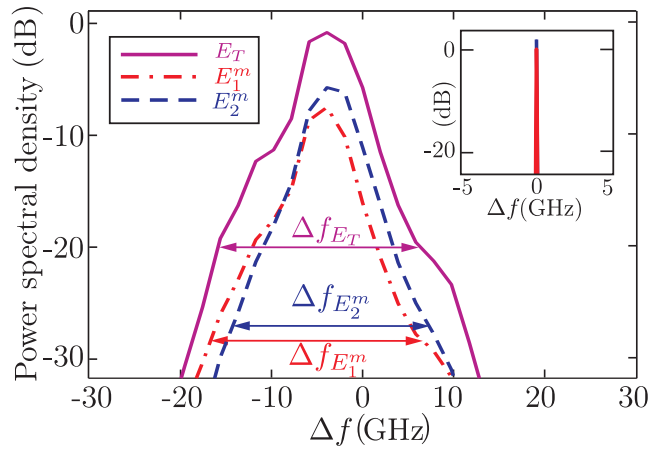

Fig. 3. (Color online) Optical spectrum of each master laser and of the transmitted signal, calculated using Welch's method. Free-running (inset) and chaotic spectra are represented. Parameters are the same than in Fig. 2 .

$\underline{\text { demultiplex chaotic optical fields with a strong spectral }}$ overlap and produced by two semiconductor lasers with identical free-running wavelength using both a coupling architecture based on APD concepts and complete chaos synchronization. Our results pave the way toward spectrally efficient optical encryption of multiple datastreams using chaotic semiconductor lasers.

The authors acknowledge the support of Fondation Supélec, of Conseil Régional de Lorraine and of National Science Foundation by ECCS Grants No. 0925713 and No. 0523923.

\section{References}

1. G.D. VanWiggeren and R. Roy, "Communication with chaotic lasers", Science 279, 1198-1200 (1998).

2. A. Argyris, D. Syvridis, L. Larger, V. Annovazzi-Lodi, P. Colet, I. Fischer, J. Garca-Ojalvo, C.R. Mirasso, L. Pesquera, and K.A. Shore, "Chaos-based communications at high bit rates using commercial fibre-optic links", Nature 437, 343-346 (2005).

3. A. Uchida, S. Kinugawa,T. Matsuura, and S. Yoshimori, "Dual synchronization of chaos in microchip lasers", Opt. Lett. 28, 19-21 (2003).

4. T. Matsuura, A. Uchida, and S. Yoshimori, "Chaotic wavelength division multiplexing for optical communication", Opt. Lett. 29, 2731-2733 (2004).

5. J.-Z. Zhang, A.-B. Wang, J.-F. Wang, and Y.-C. Wang, "Wavelength division multiplexing of chaotic secure and fiber-optic communications", Opt. Express 17, 63576367 (2009).

6. J.K. White and J.V. Moloney, "Multichannel communication using an infinite dimensional spatiotemporal chaotic system", Phys. Rev. A 59, 2422-2426 (1999)

7. J.M. Buldú, J. García-Ojalvo, and M.C. Torrent, "Multimode synchronization and communication using unidirectionally coupled semiconductor lasers", IEEE J. Quantum Electron. 40, 640-650 (2004).

8. M.W. Lee and K.A. Shore, "Chaotic message broadcasting using DFB laser diodes", Electron.Lett. 40, 614-615 (2004); ibid, "Two-mode chaos synchronisation using a multi-mode external-cavity 
laser diode and two single-mode laser diodes", IEEE J. Lightwave Technol. 23, 1068-1073 (2005).

9. L. Kocarev and U. Parlitz, "General approach for chaotic synchronization with applications to communication", Phys. Rev. Lett. 74, 5028-5031 (1995).

10. Y. Liu and P. Davis, "Dual synchronization of chaos", Phys. Rev. E 61, R2176-R2179 (2001).

11. R. Lang and K. Kobayashi, "External optical feedback effects on a semiconductor injection laser properties", IEEE J. Quantum. Electron. 16, 347-355 (1980).

12. A. Locquet, C.Masoller, and C.R. Mirasso, "Synchronization regimes of optical-feedback-induced chaos in unidirectionally coupled semiconductor lasers", Phys. Rev. E 65, 056205 (2002).

13. A. Sánchez-Díaz, C.R. Mirasso, P. Colet, and P. GarcíaFernández, "Encoded Gbit/s digital communications with synchronized chaotic semiconductor lasers", IEEE J. Quantum electron. 35, 292-297 (1999). 